\title{
Morfologia polínica de espécies de várzea e de igapó da Amazônia Central ${ }^{1}$.
}

\author{
Cleonice de Oliveira MOURA ${ }^{2}$ Maria Lúcia $\mathrm{ABSY}^{2}$, Francisco de Assis Ribeiro dos SANTOS $^{3}$, Antonio Carlos \\ MARQUES-SOUZA ${ }^{2}$
}

\section{RESUMO}

Foi estudada a morfologia dos grãos de pólen de dez espécies de plantas de várzea e igapó provenientes da Ilha da Marchantaria e Tarumã-Mirim, localizadas a $20 \mathrm{Km}$ da cidade de Manaus (AM), coletadas nos períodos de abril a agosto de 2000 e agosto de 2001. As espécies descritas foram Cassia leiandra Benth. (Caesalpiniaceae), Campsiandra comosa var. laurifolia (Benth.) Cowan (Caesalpiniaceae), Hevea spruceana (Benth.) Müll. Arg. (Euphorbiaceae), Piranhea trifoliata Baill. (Euphorbiaceae), Laetia corymbulosa Spruce ex Benth. (Flacourtiaceae), Eschweilera tenuifolia (O. Berg) Miers (Lecythidaceae), Acacia polyphylla DC. (Mimosaceae), Inga micradenia Spruce ex Benth. (Mimosaceae), Simaba orinocensis Kunth (Simarubaceae), Vitex cymosa Bert. ex Spreng. (Verbenaceae). A análise polínica constatou que estas espécies possuem grãos de pólen com características morfológicas bastante variadas.

\section{PALAVRAS-CHAVE}

Morfologia polínica, Amazônia, várzea, igapó, Ilha da Marchantaria, Tarumã-Mirim

\section{Pollen morphology of Central Amazonia floodplain and flooded forest species.}

\begin{abstract}
\section{KEY WORDS}

\section{INTRODUÇÃO}

As áreas alagáveis na Amazônia possuem um total de $300.000 \mathrm{~km}^{2}$ sendo $200.000 \mathrm{~km}^{2}$ de várzea e $100.000 \mathrm{~km}^{2}$ de igapó (Jủnk, 1993). A Amazônia é caracterizada pela extensa bacia hidrográfica e pela floresta tropical úmida (Maia, 1997). Os ambientes semi-aquáticos apresentam aproximadamente oito meses de períodos de cheia e quatro meses de períodos secos. Um aspecto importante nos ambientes de vegetação inundável é a presença de indivíduos adaptados à submersão parcial ou total durante um período prolongado (Maia, 1997).
\end{abstract}

The present study investigated the pollen grain morphology of ten flooded forest and floodplain plant species from the Marchantaria Island and Tarumã-Mirim located $20 \mathrm{Km}$ from Manaus (AM). The species were collected from April to August 2000 and in August 2001. The following species were described: Cassia leiandra Benth. (Caesalpiniaceae), Campsiandra comosa var. laurifolia (Benth.) Cowan (Caesalpiniaceae), Hevea spruceana (Benth) Müll. Arg. (Euphorbiaceae), Piranhea trifoliata Baill. (Euphorbiaceae), Laetia corymbulosa Spruce ex Benth. (Flacourtiaceae), Eschweilera tenuifolia (O. Berg) Miers (Lecythidaceae), Acacia polyphylla DC. (Mimosaceae), Inga micradenia Spruce ex Benth. (Mimosaceae), Simaba orinocensis Kunth (Simarubaceae), Vitex cymosa Bert. ex Spreng. (Verbenaceae). The pollen analysis established that these species present pollen grains of a great variability of morphological characteristics.

Pollen morphology, Amazonia, flooded forest, floodplain, Marchantaria Island, Tarumã-Mirim

Na Amazônia central, os ecossistemas inundáveis de várzea e igapó constituem-se em importantes meios para uso e preservação de recursos naturais, sendo explorados de forma sustentável. Um exemplo dessa aplicação é demonstrado no trabalho de Maia (1997), que verificou a influência e a importância da dinâmica do pulso de inundação na fisiologia, fenologia e produção de frutos de Eschweilera tenuifolia (Berg) Miers e Hevea spruceana (Benth.) Müll. Arg. Por outro lado, no trabalho palinológico pioneiro de Absy (1979) em sedimentos do Holoceno da bacia amazônica foi descrito o pólen de árvores da floresta de várzea e de igapó destacando cinco gêneros: Cassia,

${ }^{1}$ Este trabalho foi desenvolvido no âmbito do Projeto Dinâmica das Interações Bio-Ecológicas e Pulso de Inundações em Áreas Alagáveis (PPD - PPG7, No 1161/99). ${ }^{2}$ INPA, Coordenação de Pesquisas em Botânica - Caixa postal 478 - 69083-100, Manaus-AM, Brasil. E-mail: cleonice@inpa.gov.br

${ }^{3}$ UEFS-Universidade Estadual de Feira de Santana, (BA) 
Eschweilera, Hevea, Inga e Simaba. De acordo com Davis \& Heywood (1963), a morfologia do pólen é altamente significativa para auxiliar a taxonomia e ciências afins.

Neste contexto, o presente trabalho visa o estudo da morfologia polínica de dez espécies botânicas que ocorrem nas áreas de várzea da Ilha de Marchantaria, e de igapó, no rio Tarumã-Mirim, contribuindo desse modo para ampliar os estudos botânicos nessas áreas.

\section{MATERIAL E MÉTODOS}

As dez espécies estudadas foram coletadas nas florestas da várzea e de igapó, localizadas na Ilha da Marchantaria e no rio Tarumã-Mirim, respectivamente (Figura 1).

As amostras foram obtidas de ramos férteis das 10 espécies estudadas, de onde foram retirados botões florais para o preparo dos grãos de pólen. O material botânico foi incorporado ao Herbário do INPA. A preparação dos grãos de pólen para análise, foi feita baseando-se no método da acetólise (Erdtman, 1960). Após a preparação, os grãos foram montados em gelatina glicerinada e em seguida, foi feita a lutagem das lâminas com parafina. As medidas dos grãos de pólen foram feitas utilizando-se uma ocular micrometrada, adaptada a um microscópio óptico. Foram efetivadas 25 medidas dos eixos polar (P) e equatorial (E), e 10 medidas das espessuras da exina, sexina (sex) e nexina (nex), em diferentes grãos de pólen de cada espécie. As medidas compõem os cálculos estatísticos de média, desvio padrão e coeficiente de variância. Apenas a média aritmética foi calculada para as medidas de espessura da exina, nexina e sexina. As descrições morfológicas obedecem a terminologia usada por Barth \& Melhem (1988) e Punt et al. (1994).

\begin{tabular}{|l|}
\hline ABREVIATURAS UTILIZADAS \\
\hline Amb - âmbito contorno do grão em vista polar \\
NPC - número, posição e formas das aberturas \\
P/E - diâmetro polar sobre diâmetro equatorial \\
D - diâmetro \\
DM - diâmetro maior \\
Dm - diâmetro menor \\
Esp - espinho(s) \\
\hline
\end{tabular}

Os grãos de pólen foram fotomicrografados em um microscópio óptico Zeiss.

\section{RESULTADOS}

\section{Caesalpiniaceae}

- Cassia leiandra Benth. (Figura 2 A - B)

Material examinado: Amazonas, Ilha da Marchantaria (Várzea) INPA n ${ }^{0} 206.781$

Grãos de pólen médios, isopolares, forma prolata, de simetria radial, âmbito circular, 3-colporados, colpos longos sincolpados. Endoaberturas lalongadas. Exina microrreticulada; sexina mais espessa que a nexina.

Medidas: $\mathrm{P}=37,55 \pm 0,34(35-40) \mu \mathrm{m} ; \mathrm{E}=27,15 \pm$ $0,37(25-30) \mu \mathrm{m} ; \mathrm{P} / \mathrm{E}=1,38 ; \mathrm{NPC}=345 ; \mathrm{sex}=1,4 \mu \mathrm{m}$; nex $=0,74 \mu \mathrm{m} ;$ exina $=2,14 \mu \mathrm{m}$.

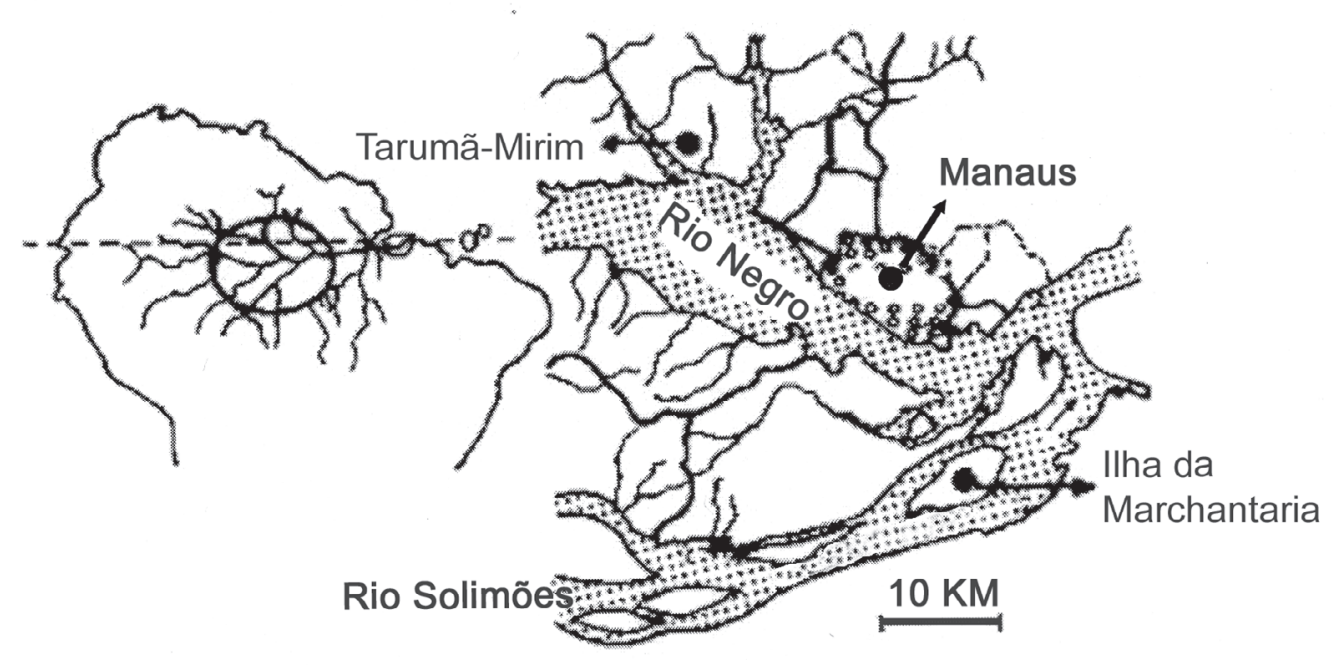

Figura 1 - Localização das áreas de estudo: Ilha da Marchantaria e do Tarumã-Mirim. Mapa adaptado de Maia (1997). 


\section{ACTA AMAZONICA}

MORFOLOGIA POLÍNICA DE ESPÉCIES DE VÁRZEA E DE IGAPÓ DA AMAZÔNIA CENTRAL
- Campsiandra comosa var. laurifolia (Benth.) Cowan ( Figura $2 \mathrm{C}-\mathrm{F}$ )

Material examinado: Amazonas, Tarumã-Mirim (Igapó) INPA $\mathrm{n}^{0} 206.774$

Grãos de pólen grandes, isopolares, forma subprolata, de simetria radial, âmbito subcircular, 3-colporados. Endoaberturas lalongadas. Exina psilada-granulada; sexina mais espessa que a nexina.

Medidas: $\mathrm{P}=51,35 \pm 0,60(47,5-57,5) \mu \mathrm{m} ; \mathrm{E}=41,45$ $\pm 0,58(37,5-45) \mu \mathrm{m} ; \mathrm{P} / \mathrm{E}=1,24 ; \mathrm{NPC}=345 ; \mathrm{sex}=1,4$ $\mu \mathrm{m} ;$ nex $=0,9 \mu \mathrm{m}$; exina $=2,3 \mu \mathrm{m}$.

\section{Euphorbiaceae}

- Hevea spruceana (Benth.) Müll. Arg. (Figura 3 A - C)

Material examinado: Amazonas, Tarumã-Mirim (Igapó) INPA $n^{0} 206772$

Grãos de pólen médios, isopolares, forma prolata, de simetria radial, âmbito subcircular, 3-colporados. Endoaberturas lalongadas. Exina microreticulada, homobrocada; sexina mais espessa que a nexina.

Medidas: $\mathrm{P}=42,6 \pm 0,87(50-35) \mu \mathrm{m} ; \mathrm{E}=31,65 \pm$ $0,55(42,5-27,5) \mu \mathrm{m}$;

$\mathrm{P} / \mathrm{E}=1,34 ; \mathrm{NPC}=345 ; \mathrm{sex}=1,35 \mu \mathrm{m} ; \mathrm{nex}=0,80 \mu \mathrm{m} ;$ exina $=2,15 \mu \mathrm{m}$.

- Piranhea trifoliata Baill. (Figura 3 D - F)

Material examinado: Amazonas, Ilha da Marchantaria (Várzea) INPA n ${ }^{0} 206779$

Grãos de pólen médios, isopolares, de simetria radial, âmbito circular, pantoporados. Exina reticulada com espinhos agudos de tamanho variado. Sexina mais espessa que a nexina.

Medidas: $\mathrm{D}=31,40 \pm 1,47(45-25) \mu \mathrm{m} ; \mathrm{NPC}=764$; sex $=1,75 \mu \mathrm{m} ;$ nex $=0,95 \mu \mathrm{m} ;$ esp $=4,0 \mu \mathrm{m} ;$ exina $=2,6 \mu \mathrm{m}$.

\section{Flacourtiaceae}

- Laetia corymbulosa Spruce ex Benth. (Figura 3 G -J)

Material examinado: Amazonas, Ilha da Marchantaria (Várzea) INPA n ${ }^{0} 208422$.

Grãos de pólen médios, isopolares, forma subprolata, de simetria radial, âmbito circular, 3-colporados. Endoaberturas circulares a lalongadas. Exina reticulada heterobrocada. Sexina mais espessa que a nexina.

Medidas: $\mathrm{P}=32,65 \pm 0,45(37,5-27,5) \mu \mathrm{m} ; \mathrm{E}=27,95$ $\pm 0,37(32,5-22,5) \mu \mathrm{m} ; \mathrm{P} / \mathrm{E}=1,22 ; \mathrm{NPC}=345 ; \mathrm{sex}=1,25$ $\mu \mathrm{m} ;$ nex $=0,75 \mu \mathrm{m} ;$ exina $=2,0 \mu \mathrm{m}$.

\section{Lecythidaceae}

- Eschweilera tenuifolia (O. Berg) Miers. (Figura 4A-D)

Material examinado: Amazonas, Tarumã-Mirim (Igapó) INPA $n^{0} 208421$
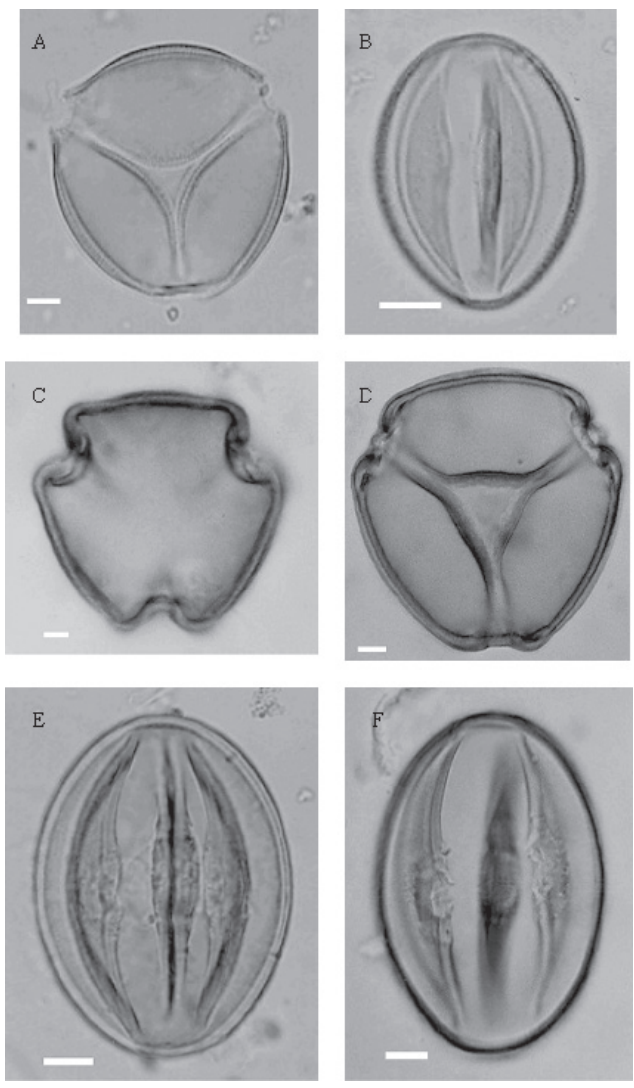

Figura 2 - Grãos de pólen das espécies A - B. Cassia leiandra Benth. (Caesalpiniaceae): A. corte óptico, vista polar. B. vista equatorial; C - D - E - F. Capsiandra comosa var. (Laurifolia Benth.) Cowam. (Caesalpiniaceae): C - D. corte óptico, vista polar. E - F. vista equatorial. Escalas: $5 \mu \mathrm{m}$.

Grãos de pólen médios, isopolares, forma subprolata, de simetria radial, âmbito triangular a circular, 3-colporados, colpos constrictos na região equatorial, endoaberturas lalongadas. Exina microrreticulada homobrocada. Sexina mais espessa que a nexina.

Medidas: $\mathrm{P}=29,15 \pm 0,31(32,5-25) \mu \mathrm{m} ; \mathrm{E}=25,05 \pm$ $0,34(27,5-22,5) \mu \mathrm{m} ; \mathrm{P} / \mathrm{E}=1,16 ; \mathrm{NPC}=345 ; \mathrm{sex}=1,5 \mu \mathrm{m}$; nex $=0,9 \mu \mathrm{m} ;$ exina $=2,4 \mu \mathrm{m}$.

\section{Mimosaceae}

- Acacia polyphylla DC. (Figura 4 E - F)

Material examinado: Amazonas, Ilha da Marchantaria (Várzea) INPA n ${ }^{0} 206778$.

Políades médias, discoidais, calimadas, radiossimétricas, compostas de 16 grãos de pólen, sendo 8 periféricos e $(4+4)$ centrais, porados. Exina psilada. Sexina mais espessa que a nexina.

Medidas: $\mathrm{DM}=37,85 \pm 0,49(45-37,5) \mu \mathrm{m} ; \mathrm{Dm}=$ $37,05 \pm 0,43(45-35) \mu \mathrm{m}$; sex $=0,82 \mu \mathrm{m} ;$ nex $=0,49 \mu \mathrm{m}$; exina $=1,31 \mu \mathrm{m}$. 


\section{ACTA AMAZONICA}
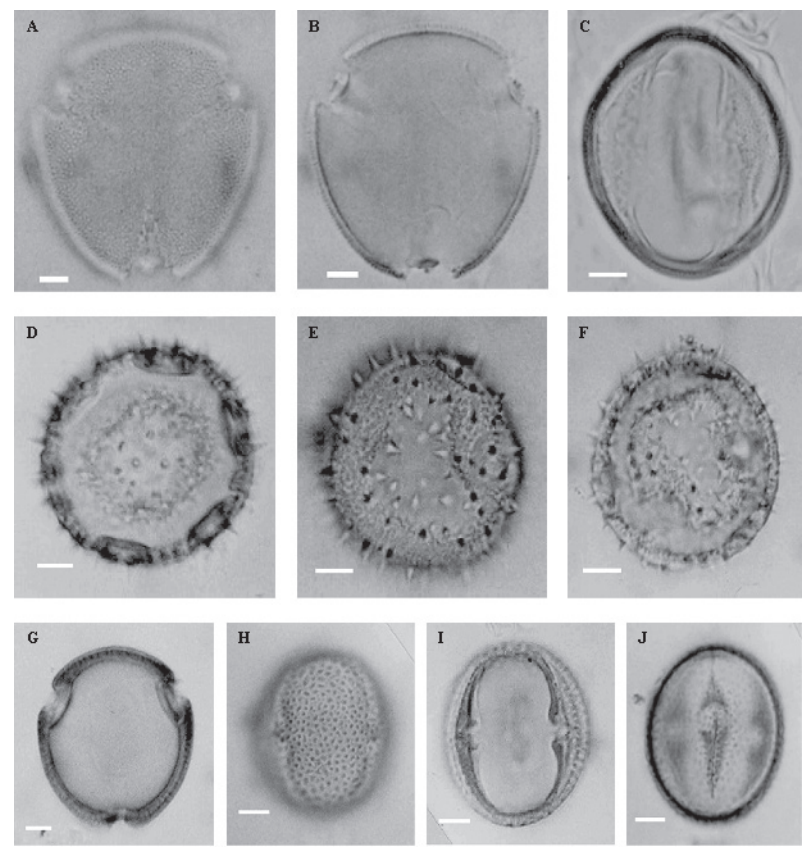

Figura 3 - Grãos de pólen das espécies A - B - C. Hevea spruceana (Benth.) Mull. Arg. (Euphorbiaceae): A - B. corte óptico, vista polar. B- C. vista equatorial; D - E - F. Piranbea trifoliata Baill. (Euphorbiaceae): corte óptico; G - H - I - J. Laetia corymbulosa Spruce ex Benth. (Flacourtiaceae): G. corte óptico, vista polar. H - I - J. vista equatorial. Escalas: $5 \mu \mathrm{m}$.

- Inga micradenia Spruce ex Benth. (Figura $4 \mathrm{G}$ - H-I)

Material examinado: Amazonas, Ilha da Marchantaria (Várzea) INPA n ${ }^{0} 206777$.

Políades muito grandes, discoidais, calimadas, radiossimétricas, compostas de 16 - 20 grãos de pólen, sendo 8 ou 10 periféricos e $(4+4$ ou $5+5)$ centrais. Exina irregularmente ornamentada, grãos porados, poros localizados na região proximal dos grãos. Sexina mais espessa que a nexina.

Medidas: $\mathrm{DM}=136,60 \pm 1,8(162-125) \mu \mathrm{m} ; \mathrm{Dm}=116,3 \pm$ $1,6(137,5-100) \mu \mathrm{m} ; \mathrm{sex}=1,9 \mu \mathrm{m} ;$ nex $=1,2 \mu \mathrm{m} ;$ exina $=3,1 \mu \mathrm{m}$.

Obs.: Estas políades são muito grandes e frágeis separando-se em grãos isolados mesmo sendo utilizado um tempo de acetólise menor.

\section{Simarubaceae}

- Simaba orinocensis Kunth (Figura 5 A - C)

Material examinado: Amazonas, Ilha da Marchantaria (Várzea) INPA no 208425.

Grãos de pólen médios, isopolares, radiossimétricos, forma prolata, âmbito subcircular, 3-colporados. Endoaberturas circulares. Exina microrreticulada, homobrocada. Sexina mais espessa que a nexina.
Medidas: $\mathrm{P}=23,75 \pm 0,27(27,5-20) \mu \mathrm{m} ; \mathrm{E}=15,6 \pm$ $0,32(12,5-20) \mu \mathrm{m} ; \mathrm{P} / \mathrm{E}=1,52 ; \mathrm{NPC}=345 ; \mathrm{sex}=0,89 \mu \mathrm{m}$; nex $=0,60 \mu \mathrm{m} ;$ exina $=1,5 \mu \mathrm{m}$.

\section{Verbenaceae}

- Vitex cymosa Bert. ex Spreng. (Figura 5 D - G)

Material examinado: Amazonas, Ilha da Marchantaria (Várzea) INPA n ${ }^{\circ} 208423$.

Grãos de pólen médios, isopolares, radiossimétricos, forma prolata esferoidal, 3-colporados, colpos com membrana granulada; âmbito circular. Endoaberturas pouco distintas. Exina reticulada, homobrocada. Sexina mais espessa que a nexina.

Medidas: $\mathrm{P}=26,75 \pm 0,39(30-25) \mu \mathrm{m} ; \mathrm{E}=24,65 \pm$ $0,41(25-17,5) \mu \mathrm{m} ; \mathrm{P} / \mathrm{E}=1,08 ; \mathrm{NPC}=345 ; \mathrm{sex}=1,6 \mu \mathrm{m}$; nex $=0,85 \mu \mathrm{m}$; exina $=2,4 \mu \mathrm{m}$.

\section{DISCUSSÃO E CONCLUSÃO}

As áreas de várzea da Amazônia central apresentam maior diversidade de espécies arbóreas do que as áreas de igapó. De acordo com Ducke \& Black (1953), na Amazônia, a variação da distribuição de chuvas durante o ano proporciona períodos de floração e frutificação diferentes para cada região.
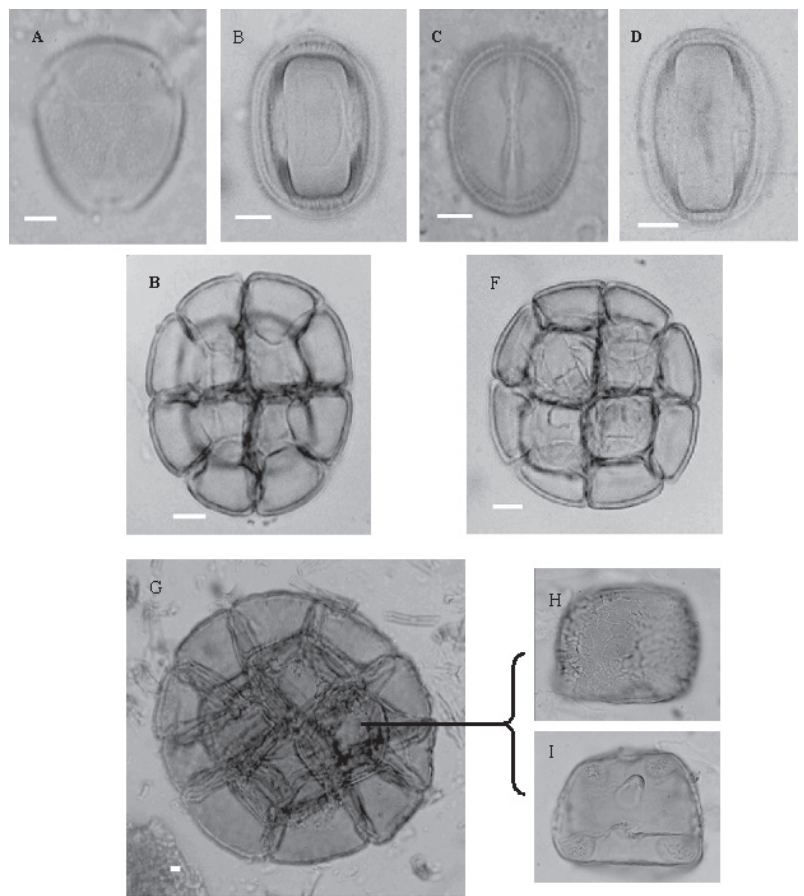

Figura 4 - Grãos de pólen das espécies A - B - C - D. Eschweilera tenuifolia (O. Berg) Miers. (Lecythidaceae): A. corte óptico, vista polar. B - C - D. Vista Equatorial; E - F. Acacia polyphylla DC, corte óptico; G - H - I. Inga micradenia Spruce ex Benth. (Mimosaceae): G. corte óptico; H - I. grãos isolados da políade. Escalas: $5 \mu \mathrm{m}$. 


\section{ACTA AMAZONICA}
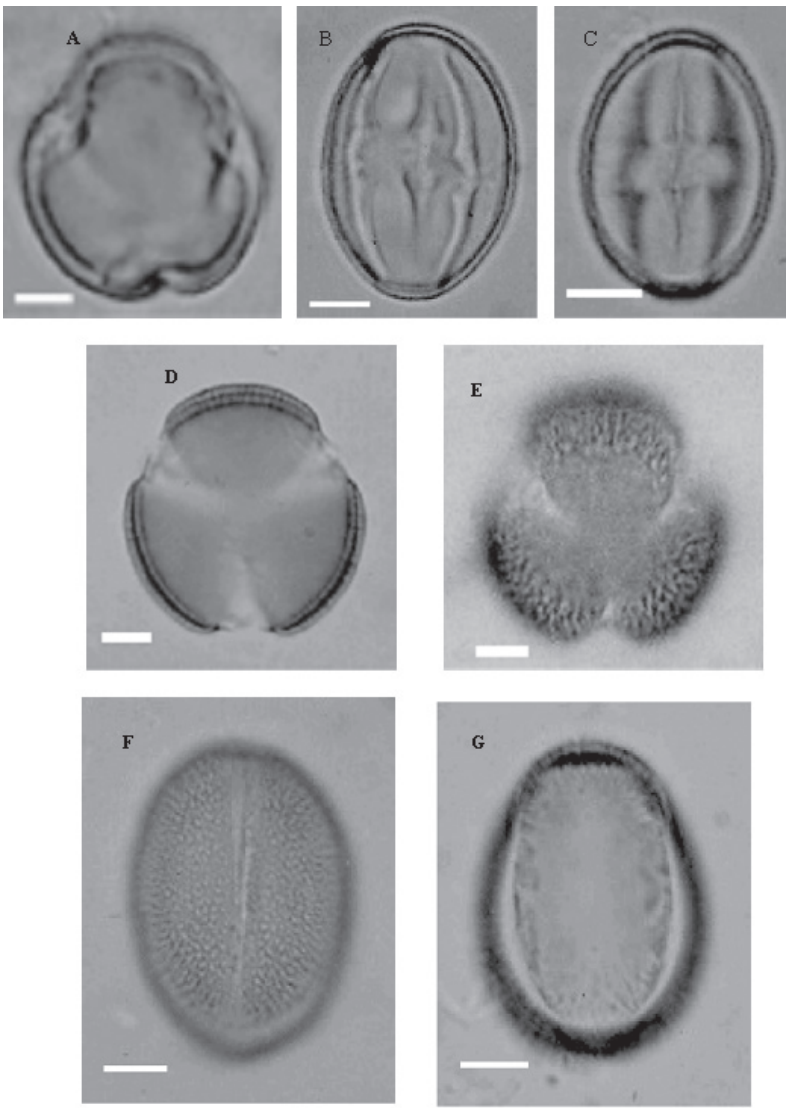

Figura 5 - Grãos de pólen das espécies A- B- C. Simaba orinocensis Kunth.(Simarubaceae): A. corte óptico, vista polar. B- C. vista equatorial. D- E- F- G. Vitex cymosa Bert. ex Spruce (Verbenaceae): D- E. corte óptico, vista polar. F- G. vista equatorial. Escalas: $5 \mu \mathrm{m}$.

Dos gêneros correspondentes às espécies deste trabalho, Absy (1979) fez a descrição da morfologia dos grãos de pólen de Cassia, Hevea, Eschweilera, Inga e Simaba, estudadas em amostras de sedimentos da bacia amazônica. Recentemente, Carreira et al. (1996) descreveram o pólen de outras espécies dos gêneros Cassia, Campsiandra, Acacia e Inga, estudadas em amostras recente da Amazônia brasileira. Esses gêneros foram escolhidos de amostras disponíveis nos herbários MG (Museu Paraense Emílio Goeldi), IAN (CPATU/EMBRAPA) e INPA (Instituto Nacional de Pesquisas da Amazônia).

Os resultados das análises morfológicas dos grãos de pólen das espécies estudadas mostraram que as características morfológicas a nível genérico são semelhantes às dos gêneros descritos pelos autores acima citados. No presente trabalho, no entanto, foram descritos os grãos de pólen de duas espécies de dois gêneros diferentes da família Euphorbiaceae: Piranbea trifoliata e Hevea spruceana. Essas duas espécies apresentaram características morfológicas bastante distintas: os grãos de pólen de Piranbea trifoliata apresentaram a exina reticulada e com espinhos, enquanto que a exina de Hevea spruceana é microrreticulada e sem espinhos.

\section{AGRADECIMENTOS.}

Agradecemos o apoio financeiro recebido através do Instituto Nacional de Pesquisas da Amazônia (INPA) e do Projeto Dinâmica das Interações Bio-Ecológicas e Pulso de Inundações em Áreas Alagáveis (PPD - PPG7, No 1161/99); à Dra. M. Gercília M. Soares, pela cooperação e sugestões; à Ana Rita Silva, pela ajuda sempre quando solicitada.

\section{BIBLIOGRAFIA CITADA}

Absy, M.L. 1979. Palynological study of Holocene sediments in the Amazon Basin. Thesis, University of Amsterdam, 102pp.

Barth, O.M; Melhem, T.S. 1988. Glossário ilustrado de Palinologia. Ed. UNICAMP, Campinas, 75pp.

Carreira, L.M.M.; Silva, M. F.; Lopes, J.R.C.; Nascimento, L.A.S. 1996. Catálogo de Pólen das Leguminosas da Amazônia Brasileira. Museu Paraense Emílio Goeldi, Belém, 137pp.

Davis, P. H.; Heywood, V. H. 1963. Principles of Angiosperm Taxonomy. Oliver Boyd, London, 556pp.

Ducke, A.; Black, G. A. 1953. Notas sobre a fitogeografia da Amazônia brasileira. Bol. Téc. Inst. Agron, Norte, 29: 1-62.

Erdtman, G. 1960. The acetolysis method: in a revised description. Sv. Bot. Tidskr. Lund., 54(4):561-564.

Junk, W. J. 1993. Wetlands of tropical South America. In: Whigham, D.; Hejny, S.; Dykyjova, D. (eds.) Wetlands in the Amazon floodplain. Hidrobiologia, 263:155-162.

Maia, L. M., 1997. Influência do Pulso de Inundação na Fisiologia, Fenologia e Produção de Frutos de Hevea spruceana (Euphorbiaceae) $e$ Eschweilera tenuifolia (Lecythidaceae), em áreas inundáveis de igapó da Amazônia Central. Tese de Doutorado Instituto Nacional de Pesquisas da Amazônia/ Universidade Federal do Amazonas, 186pp.

Punt, W.; Blackmore, S.; Nilsson, S.; Le Thomas, A. 1994. Glossary of Pollen and Spore Terminology. LPP Foundation. Utrecht Contributions Series n. 1, Utrecht, 71pp. 
